\title{
Analytical technique for neutral delay differential equations with proportional and constant delays
}

\author{
Normah Maan ${ }^{\mathrm{a}, *}$, Aminu Barde $\mathrm{a}^{\mathrm{a}, \mathrm{b}}$ \\ ${ }^{a}$ Department of Mathematical Sciences, Universiti Teknologi, 81310 Skudai, Johor, Malaysia. \\ ${ }^{b}$ Department of Mathematical Sciences, Abubakar Tafawa Balewa University, Bauchi, Nigeria.
}

\begin{abstract}
Neutral delay differential equations (NDDEs) are a type of delay differential equations (DDEs) that arise in numerous areas of applied sciences and play a vital role in mathematical modelling of real-life phenomena. Some techniques have experienced difficulties in finding the approximate analytical solution which converges rapidly to the exact solution of these equations. In this paper, an analytical approach is proposed for solving linear and nonlinear NDDEs with proportional and constant delays based on the homotopy analysis method (HAM) and natural transform method where the nonlinear terms are simply calculated as a series of, He's polynomial. The proposed method produces solutions in the form of a rapidly convergent series which leads to the exact solution from only a few numbers of iterations. Some illustrative examples are solved, and the convergence analysis of the proposed techniques was also provided. The obtained results reveal that the approach is very effective and efficient in handling both linear and nonlinear NDDEs with proportional and constant delays and can also be used in various types of linear and nonlinear problems.
\end{abstract}

Keywords: Neutral delay differential equations, He's polynomial, natural transform method, homotopy analysis method.

2010 MSC: 65L03, 34K99.

(C)2020 All rights reserved.

\section{Introduction}

Delay differential equation is a branch of functional differential equation arising in several applications from various fields of studies, for instances biology, population dynamics, chemistry, physics, control theory, and many others $[2,3,7,14,16,20,21,24,25]$. Unlike in the case of ordinary differential equations (ODEs) the unknown functions in DDEs depend not only on the present values of derivatives of independent variables, but also depend on the values of the functions at some previous times. For this reason, DDEs particularly NDDEs provide an efficient mathematical tool to model various phenomena from reallife problems. Recently, there is a rapid development of new analytical methods with the aim to obtain the solution of different classes of DDEs. However, it is obvious that a lot of these introduced techniques have encountered some difficulties in finding approximate analytical solutions which converge to an exact

\footnotetext{
${ }^{*}$ Corresponding author

Email address: normahmaan@utm.my (Normah Maan)
}

doi: $10.22436 /$ jmcs.020.04.07 
solution of DDEs more specifically NDDEs. It is therefore very difficult to solve NDDEs with proportional and constant delays analytically due to their special transcendental nature. Hence, they are mostly solved by numerical methods $[8,26,27,29]$. Therefore, it is of more interest to develop a new analytical approach for such equations.

HAM is a powerful technique introduced by Lio [11] for solving different types of linear and nonlinear problems $[1,12,13,19,22]$. Therefore, in this article, we introduced a new analytical approach from the combination of HAM and Natural transform method for both linear and nonlinear NDDEs with proportional and constant delays. Based on this technique the nonlinear terms of NDDEs would be computed as a series of He's polynomial. The method gives solution in a series form which converges to an exact solution using a few numbers of terms. In addition, some important theorems were established in order to guarantee the convergence of the new approach and the maximum estimated error between the approximate and the exact solutions.

This paper is organized as follows. In Section 2 the definitions and concept of Natural transform for NDDEs are given. The analysis of the technique is presented in Section 3. The convergence analysis of the proposed method was given in Section 4. To show the effectiveness of the new approach solutions to a different form of NDDEs was rendered in Section 5. Finally, the conclusion follows in Section 6.

\section{Natural transform method}

Khan and Khan [10] introduced an integral transform called N-transform, and it was later renamed as natural transform by Belgacem and Silambarasan [4]. This transform was derived from the renowned Fourier integral and converged to Laplace and Sumudu transforms depending on the values of the transform variables. The basic concepts of natural transform that are useful in this paper are rendered below.

Definition $2.1([6])$. Let $t \in(-\infty, \infty)$, then the natural transform of the function $f(t)$ is defined by

$$
\mathbf{N}^{+}[f(t)]=R^{+}(s, u)=\int_{-\infty}^{\infty} e^{-s t} f(u t) d t ; \quad s, u \in(0, \infty]
$$

where $\mathbf{N}^{+}$denotes as natural transform and $s, u$ are transforming variables.

Now, (2.1) can be written in the following form [6, 23]

$$
\begin{aligned}
\mathbf{N}^{+}[f(t)]=R^{+}(s, u) & =\int_{-\infty}^{\infty} e^{-s t} f(u t) d t ; \quad s, u \in(0, \infty] \\
& =\int_{-\infty}^{0} e^{-s t} f(u t) d t ; s, u \in(-\infty, 0)+\int_{0}^{\infty} e^{-s t} f(u t) d t ; s, u \in(0, \infty) \\
& =\mathbf{N}^{-}[f(t)]+\mathbf{N}^{+}[f(t)] \\
& =\mathbf{N}[\mathbf{f}(t) H(-t)]+\mathbf{N}[f(t) H(t)] \\
& =R^{-}(s, u)+R^{+}(s, u) .
\end{aligned}
$$

Here, $H($.$) is the Heaviside function. Suppose the function f(t) H(t)$ is defined on $\mathbb{R}^{+}$and $t \in \mathbb{R}$ then we define the natural transform over the set

$$
A=\left\{f(t): \exists M, \tau_{1}, \tau_{2}>0,|f(t)|<M e^{\frac{|t|}{\tau_{j}}}, t \in(-1)^{j} \times[0, \infty), j \in \mathbb{Z}^{+}\right\}
$$

as in the given integral:

$$
\mathbf{N}^{+}[\mathrm{f}(\mathrm{t})]=\mathrm{R}^{+}(\mathrm{s}, \mathrm{u})=\int_{0}^{\infty} e^{-s t} f(u t) d t ; \quad s, u \in(0, \infty] .
$$


Theorem $2.2([4])$. Let $f(t)=\sum_{n=0}^{\infty} a_{n} t^{n}$, then the generalized natural transform of the function $f(t)$ is given as

$$
\mathbf{N}^{+}[f(t)]=R^{+}(s, u)=\sum_{n=0}^{\infty} \frac{n ! a_{n} u^{n}}{s^{n+1}}
$$

where $a_{n} \in \mathbb{N}$.

Property 2.1 ([6]). Let a be a non-zero constant and $\mathrm{f}(\mathrm{at}) \in \mathrm{A}$, then

$$
\mathbf{N}^{+}[\mathrm{f}(\mathrm{at})]=\frac{1}{\mathrm{a}} \mathrm{R}\left(\frac{\mathrm{s}}{\mathrm{a}}, \mathrm{u}\right) .
$$

Theorem 2.3 ([5]). If $\mathrm{H}_{\tau}$ is the Heaviside function for any real number $\tau \geqslant 0$ defined as

$$
\mathrm{H}_{\tau}(\mathrm{t})= \begin{cases}1, & \text { for } \mathrm{t} \geqslant \tau \\ 0, & \text { for } \mathrm{t}<\tau,\end{cases}
$$

then the natural transform of the shifted function $f(t-\tau)=f(t-\tau) H_{\tau}(t)$ is given by

$$
\mathbf{N}^{+}\left[f(t-\tau) H_{\tau}(t)\right]=e^{\frac{-s \tau}{u}} N^{+}[f(t)]
$$

Theorem 2.4 ([4]). Let $\mathrm{f}^{(\mathrm{n})}(\mathrm{t})$ be the $\mathrm{n}^{\text {th }}$ derivatives of the function $\mathrm{f}(\mathrm{t})$ then its natural transform is given by

$$
\mathbf{N}^{+}\left[f^{n}(t)\right]=R_{n}^{+}(s, u)=\frac{s^{n}}{u^{n}} R(s, u)-\sum_{k=1}^{n} \frac{s^{n-k}}{u^{(n-k)+1}} f^{k-1}(0) .
$$

Corollary 2.5. Let $\mathbf{N}^{+}[\mathrm{f}(\mathrm{at})]=\mathrm{R}^{+}(\mathrm{as}, \mathrm{u})$, if $\mathrm{f}^{(\mathrm{n})}(\mathrm{at})$ is the $\mathrm{n}^{\text {th }}$ derivative of the function $\mathrm{f}(\mathrm{at})$ with respect to $t$, then its natural transform is given by

$$
\mathbf{N}^{+}\left[f^{n}(a t)\right]=R_{n}^{+}(a s, u)=\frac{s^{n}}{(a u)^{n}} R^{+}(a s, u)-\sum_{k=1}^{n} \frac{s^{n-k}}{(a u)^{(n-k)+1}} f^{(k-1)}(0) .
$$

Proof. For $n=1$ and $n=2$, (2.2) gives the natural transform of first and second derivatives of $f(a t)$, respectively.

$$
\begin{aligned}
& \mathbf{N}^{+}\left[f^{\prime}(a t)\right]=R_{1}^{+}(a s, u)=\frac{s}{a u} R^{+}(a s, u)-\frac{f(0)}{a u}, \\
& \mathbf{N}^{+}\left[f^{\prime \prime}(a t)\right]=R_{2}^{+}(a s, u)=\frac{s^{2} R^{+}(a s, u)-s f(0)}{(a u)^{2}}-\frac{f^{\prime}(0)}{a u} .
\end{aligned}
$$

Then by induction suppose the condition holds for $n$, then it is suffices to show it also holds for $n+1$. Now from (2.3) we have

$$
\begin{aligned}
\mathbf{N}^{+}\left[f^{(n+1)}(a t)\right] & =\mathbf{N}^{+}\left[\left(f^{(n)}(a t)\right)^{\prime}\right]=R_{n+1}^{+}(a s, u)=\frac{s}{a u} R_{n}^{+}(a s, u)-\frac{f^{(n)}(0)}{a u} \\
& =\frac{s}{a u}\left[\frac{s^{n}}{(a u)^{n}} R^{+}(a s, u)-\sum_{k=1}^{n} \frac{s^{n-k}}{(a u)^{(n-k)+1}} f^{(k-1)}(0)\right]-\frac{f^{(n)}(0)}{a u} \\
& =\frac{s^{n+1}}{(a u)^{n+1}} R^{+}(a s, u)-\sum_{k=1}^{n+1} \frac{s^{(n-k)+1}}{(a u)^{(n-k)+2}} f^{(k-1)}(0)
\end{aligned}
$$

Which holds for $n+1$ and hence the result. 
Corollary 2.6. If $\mathrm{f}^{(\mathrm{n})}(\mathrm{t}-\tau)$ is $\mathrm{n}^{\text {th }}$ derivative of the shifted function $\mathrm{f}(\mathrm{t}-\tau)$ with respect to $\mathrm{t}$, then its natural transform is given by

$$
\mathbf{N}^{+}\left[f^{n}(t-\tau)\right]=e^{\left(-\frac{s \tau}{u}\right)} R_{n}^{+}(s, u)=\frac{s^{n}}{u^{n}} e^{\left(-\frac{s \tau}{u}\right)} R^{+}(s, u)-\sum_{k=1}^{n} \frac{s^{n-k}}{(u)^{(n-k)+1}}\left[\lim _{t \rightarrow 0} f^{(k-1)}(t-\tau)\right] .
$$

Proof. The proof of Corollary 2.6 is similar to that of Corollary 2.5, so for $n=1$ and $n=2$ in (2.4) we respectively have the natural transform of first and second derivatives of $f(t-\tau)$.

$$
\begin{aligned}
& \mathbf{N}^{+}\left[f^{\prime}(t-\tau)\right]=\frac{s}{u} e^{\frac{-s \tau}{u}} R^{+}(s, u)-\frac{1}{u} \lim _{t \rightarrow 0} f(t-\tau), \\
& \mathbf{N}^{+}\left[f^{\prime \prime}(t-\tau)\right]=\frac{s^{2} e^{\frac{-s \tau}{u}} R^{+}(s, u)-s \lim _{t \rightarrow 0} f(t-\tau)}{u^{2}}-\frac{\lim _{t \rightarrow 0} f^{\prime}(t-\tau)}{u} .
\end{aligned}
$$

Also by induction assume (2.4) is true for $n$ and we also have to prove for $n+1$. From (2.5) we can have

$$
\begin{aligned}
\mathbf{N}^{+}\left[f^{(n+1)}(t-\tau)\right] & =\mathbf{N}^{+}\left[\left(f^{n}(t-\tau)\right)^{\prime}\right]=\frac{s}{u} \mathbf{N}^{+}\left[f^{(n)}(t-\tau)\right]-\frac{\lim _{t \rightarrow 0} f^{n}(t-\tau)}{u} \\
& =\frac{s}{u}\left[\frac{s^{n}}{u^{n}} e^{\frac{-s \tau}{u}} R^{+}(s, u)-\sum_{k=1}^{n} \frac{s^{n-k}}{u^{(n-k)+1}}\left[\lim _{t \rightarrow 0} f^{(k-1)}(t-\tau)\right]\right]-\frac{\lim _{t \rightarrow 0} f^{(n)}(t-\tau)}{u} \\
& =\frac{s^{n}}{u^{n}} e^{\frac{-s \tau}{u}} R^{+}(s, u)-\sum_{k=1}^{n+1} \frac{s^{n-k+1}}{u^{(n-k)+2}}\left[\lim _{t \rightarrow 0} f^{(k-1)}(t-\tau)\right] .
\end{aligned}
$$

Which is true for $n+1$ and hence the result. For more application of natural transform in solving different type of differential and integral equations see $[5,15,17,18,23]$.

\section{Analysis of the method}

Consider the following n-order Neutral DDEs of the form

$$
[v(t)+v(\beta(t))]^{(n)}=F\left[t, v^{k}, v^{k}\left(\beta_{i}(t)\right)\right], \quad t \in[0, d],
$$

where $i=1,2, \ldots, r, k=1,2, \ldots, n-1, \beta_{i}(t)$ are delay functions such that $v(\beta(t))=\max \left[\beta_{i}(t)\right]$ subject to the given initial condition

$$
v^{\mathrm{k}}(0)=v_{0}^{\mathrm{k}}, v(\mathrm{t})=\mathrm{p}(\mathrm{t}), \mathrm{t}<0 .
$$

In this paper two types of delays $\beta_{\mathfrak{i}}(t)$ are to be considered:

Case I. $\beta_{\mathfrak{i}}(t)=a_{\mathfrak{i}} t$, where $a_{\mathfrak{i}} \in(0,1)$ (proportional delay);

Case II. $\beta_{i}(t)=t-\tau_{i}$, where $\tau_{i}>0$ is a real constant (constant delay).

Now (3.1) can be written in the following form

$$
\mathrm{L}(v+v(\beta))+\mathrm{R}(v)+\mathrm{F}(v)=\mathrm{g}(\mathrm{t})
$$

with specified initial conditions. The linear terms are split into $L+R$ where $L$ is the highest order bounded linear operator, $R$ is the remaining of the linear operator which is also bounded, and $F$ is a continuous function satisfies the Lipschitz condition with Lipschitz constant $\alpha \in[0, d]$, represents the non-linear term. That is, there exists some positive numbers $\alpha_{1}, \alpha_{2}$ and $\alpha$ such that

$$
\left\{\|\mathrm{L}(v)\| \leqslant \alpha_{1}\|v(\mathrm{t})\|,\|\mathrm{R}(v)\| \leqslant \alpha_{2}\|v(\mathrm{t})\|,|\mathrm{f}(v)-\mathrm{f}(\mathrm{u})| \leqslant \alpha|v-u|, \forall \mathrm{t} \in[0, \mathrm{~d}]\right\} .
$$


Take the natural transform of both sides of (3.3) we obtain:

$$
\mathbf{N}^{+}\left[\mathrm{L}\left(v+v_{\beta}\right)\right]+\mathbf{N}^{+}[\mathrm{R}(v)+\mathrm{F}(v)-\mathrm{g}(\mathrm{t})]=0 .
$$

Substitute (3.2) into (3.4), and simplify using the differential properties of natural transform to obtain the following with respect to two types of delays defined in Case I and Case II, respectively.

$$
\begin{aligned}
& \mathbf{N}^{+}\left[v(t)+\frac{1}{a^{n}} v(a t)\right]-\sum_{k=1}^{n}\left(1+\frac{1}{a^{(n+1-k)}}\right) \frac{u^{n-k}}{s^{k}} v^{k-1}(0) \\
& \quad+\frac{u^{n}}{s^{n}} \mathbf{N}^{+}[R(v)+F(v)-g(t)]=0, \\
& \mathbf{N}^{+}\left[v(t)+e^{\frac{-s \tau}{u}} v(t)\right]-\sum_{k=1}^{n} \frac{u^{n-k}}{s^{k}}\left[v^{k-1}(0)+\lim _{t \rightarrow 0} v^{k-1}(t-\tau)\right] \\
& +\frac{u^{n}}{s^{n}} \mathbf{N}^{+}[R(v)+F(v)-g(t)]=0,
\end{aligned}
$$

where $a=\max \left[a_{i}\right]$ and $\tau=\max \left[\tau_{i}\right]$.

Now, from (3.5) we can define the following nonlinear operators

$$
\begin{aligned}
\mathrm{N}[\phi(t ; q)]= & \mathbf{N}^{+}\left[\phi(t ; q)+\frac{1}{a^{n}} \phi(a t ; q)\right]-\sum_{k=1}^{n}\left(1+\frac{1}{a^{(n+1-k)}}\right) \frac{u^{n-k}}{s^{k}} \phi^{k-1}(0) \\
& +\frac{u^{n}}{s^{n}} \mathbf{N}^{+}[R(\phi(t ; q))+F(\phi(t ; q))-g(t)], \\
N[\phi(t ; q)]= & \mathbf{N}^{+}\left[\phi(t ; q)+e^{\frac{-s \tau}{u}} \phi(t ; q)\right]-\sum_{k=1}^{n} \frac{u^{n-k}}{s^{k}}\left[\phi^{k-1}(0)\right. \\
& \left.+\lim _{t \rightarrow 0} \phi^{k-1}(t ; q-\tau)\right]+\frac{u^{n}}{s^{n}} \mathbf{N}^{+}[R(\phi(t ; q))+F(\phi(t ; q))-g(t)],
\end{aligned}
$$

where $\mathrm{q} \in[0,1]$ is an embedding parameter, $\phi(\mathrm{t} ; \mathrm{q})$ is a function of real variables $\mathrm{t}$ and $\mathrm{q}$. Then by means of HAM we construct the homotopy equation as

$$
(1-q) \mathbf{N}^{+}\left[\phi(t ; q)-v_{0}(t)\right]=h q H(t) N[\phi(t ; q)],
$$

where $\mathbf{N}^{+}$denotes natural transform, $h \neq 0$ is auxiliary parameter, $H(t) \neq 0$ is auxiliary function, $v_{0}(t)$ is initial approximation of $v(t)$, and $\phi(t ; q)$ is unknown function. From (3.7) when $q=0$ and $q=1$ we respectively have the following equations

$$
\phi(t, 0)=v_{0}(t), \quad \phi(t, 1)=v(t) .
$$

Thus, as $q$ increases from 0 to 1 , the solution $\phi(t, q)$ varies from the initial approximation $v_{0}(t)$ to the exact solution $v(t)$. In topology such kind of variation is called deformation and (3.7) is called zero-order deformation equation. Expanding $\phi(t ; q)$ in Taylor series with respect to $q$ we have

$$
\phi(t, q)=\phi(t, 0)+\sum_{m=1}^{\infty} v_{m}(t) q^{m}
$$

where

$$
v_{m}(t)=\left.\frac{1}{m !} \frac{\partial^{m} \phi(t ; q)}{\partial q^{m}}\right|_{q=0}
$$


Suppose that the initial approximation $v_{0}(t)$, auxiliary parameter $h$ and the auxiliary function $H(t)$ are appropriately chosen so that the series in (3.8) converges at $q=1$, that is

$$
\phi(t, 1)=v_{0}(t)+\sum_{m=1}^{\infty} v_{m}(t)
$$

Now define vector

$$
\vec{v}_{n}(t)=\left[v_{0}(t), v_{1}(t), \ldots, v_{n}(t)\right] .
$$

By differentiate (3.7) $m$ times with respect to $q$ and setting $q=0$ and finally divided by $m$ ! we obtain the so-called $\mathrm{m}^{\text {th }}$-order deformation equation

$$
\mathbf{N}^{+}\left[v_{m}(t)-\chi_{m} v_{m-1}(t)\right]=h H(t) R_{y, m}\left[\vec{v}_{m-1}(t)\right],
$$

where

$$
R_{y, m}\left[\vec{v}_{m-1}(t)\right]=\left.\frac{1}{(m-1) !} \frac{\partial^{m-1} N[\phi(t, q)]}{\partial q^{m-1}}\right|_{q=0}
$$

and

$$
\chi_{m}= \begin{cases}0, & m \leqslant 1 \\ 1, & m>1 .\end{cases}
$$

Now, taking the inverse natural transform on both sides of (3.10) we have

$$
v_{m}(t)=\chi_{m} v_{m-1}(t)+h N^{-}\left[H(t) R_{y, m}\left[\vec{v}_{m-1}(t)\right]\right] .
$$

Therefore, $v_{m}(t)$ for $m \geqslant 1$ can be easily obtained from (3.12), and at $M^{\text {th }}$ order we have

$$
v(t)=\sum_{m=0}^{M} v_{m}(t)
$$

Therefore, as $M \rightarrow \infty$ we obtained the following recursive relations of (3.1) and (3.2) with respect to type of delays defined in Case I and Case II, respectively.

$$
\begin{aligned}
v_{m}(t)= & \left(\chi_{m}+h\right) v_{m-1}(t)+\frac{1}{a^{n}} h v_{m-1}(a t)-h\left(1-\chi_{m}\right) \mathbf{N}^{-}\left[\sum_{k=1}^{n}\left(1+\frac{1}{a^{n-k+1}}\right)\right. \\
& \left.\times \frac{u^{k-1}}{s^{k}} v^{k-1}(0)\right]+h \mathbf{N}^{-}\left\{\frac{u^{n}}{s^{n}} \mathbf{N}^{+}\left[R\left(v_{m-1}(t)\right)+H_{m-1}\left(v_{0}, v_{1} \ldots v_{n}\right)-g\right]\right\}, \\
v_{m}(t)= & \left(\chi_{m}+h\right) v_{m-1}(t)+h v_{m-1}(t-\tau)-h\left(1-\chi_{m}\right) \mathbf{N}^{-}\left[\sum _ { k = 1 } ^ { n } \frac { u ^ { k - 1 } } { s ^ { k } } \left[v^{k-1}(0)\right.\right. \\
& \left.\left.+\lim _{t \rightarrow 0} v^{k-1}(t-\tau)\right]\right]+h \mathbf{N}^{-}\left\{\frac{u^{n}}{s^{n}} \mathbf{N}^{+}\left[R\left(v_{m-1}(t)\right)+H_{m-1}\left(v_{0}, . . v_{n}\right)-g\right]\right\} .
\end{aligned}
$$

For $m \geqslant 1$, the nonlinear operators $F(v)$ are expanded as series of He's polynomials $H_{m-1}\left(v_{0}, v_{1} \ldots v_{n}\right)$ define by

$$
H_{m}\left(v_{0}, v_{1}, \ldots, v_{n}\right)=\left.\frac{1}{m !} \frac{\partial^{m}}{\partial q^{m}} F\left(\sum_{p=0}^{m} q^{p} v_{p}\right)\right|_{q=0} .
$$

\section{Convergence theorem and estimated error}

In this section the convergence analysis and estimated error of our derived method will be presented.

Theorem 4.1. Suppose the homotopy series (3.9) converges, then it must be the solution of original problem defined in (3.1) and (3.2), where $v_{m}(t)$ is governed by the higher order deformation (3.10) under the definitions in equations (3.6) and (3.11). 
Proof. If the series $\sum_{m=0}^{\infty} v_{m}(t)$ converges, then

$$
\lim _{m \rightarrow \infty} v_{m}(t)=0 \text {. }
$$

Let $S(t)=\sum_{m=0}^{\infty} v_{m}(t)$, then it suffices to show that $S(t)$ satisfies (3.3). From definition of $\chi_{m}$ we have

$$
\sum_{m=1}^{n}\left[v_{m}(t)-\chi_{m} v_{m-1}(t)\right]=v_{1}+\left(v_{2}-v_{1}\right)+\left(v_{3}-v_{2}\right)+\cdots+\left(v_{n}-v_{n-1}\right)=v_{n} \text {. }
$$

From (4.2) we obtained

$$
\sum_{m=1}^{\infty}\left[v_{m}(t)-\chi_{m} v_{m-1}(t)\right]=\lim _{n \rightarrow \infty} \sum_{m=1}^{n}\left[v_{m}(t)-\chi_{m} v_{m-1}(t)\right]=\lim _{n \rightarrow \infty} v_{n}(t)=0 .
$$

From (4.1) we have

$$
\sum_{m=1}^{\infty}\left[v_{m}(t)-\chi_{m} v_{m-1}(t)\right]=\lim _{n \rightarrow \infty} \sum_{m=1}^{n}\left[v_{m}(t)-\chi_{m} v_{m-1}(t)\right]=\lim _{n \rightarrow \infty} v_{n}(t)=0 .
$$

According to (3.10) and (4.3) we have

$$
\sum_{m=1}^{\infty} N^{+}\left[v_{m}(t)-\chi_{m} v_{m-1}(t)\right]=\hbar H(t) \sum_{m=1}^{\infty} R_{m}\left(\vec{v}_{m-1}(t)\right)=0 .
$$

Since $\hbar \neq 0$ and $H(t) \neq 0$ therefore

$$
R_{m}\left(\vec{v}_{m-1}(t)\right)=0 .
$$

According to (3.6) and (3.11) we have to consider the two types of delay defined in Case I and Case II.

Case I. (Proportional delay):

$$
\begin{aligned}
\mathrm{R}_{\mathrm{m}}\left(\vec{v}_{\mathrm{m}-1}(\mathrm{t})\right)= & \sum_{\mathrm{m}=1}^{\infty} \frac{1}{(\mathrm{~m}-1) !} \frac{\partial^{\mathrm{m}-1}}{\partial \mathrm{q}^{\mathrm{m}-1}} \mathbf{N}^{+}\left[\phi(\mathrm{t} ; \mathrm{q})+\frac{1}{\mathrm{a}^{\mathrm{n}}} \phi(\mathrm{at} ; \mathrm{q})\right] \\
& -\mathbf{N}^{+}\left[\sum_{\mathrm{k}=1}^{\mathrm{n}}\left(1+\frac{1}{\mathrm{a}^{(\mathrm{n}+1-\mathrm{k})}}\right) \frac{\mathrm{u}^{\mathrm{n}-\mathrm{k}}}{\mathrm{s}^{\mathrm{k}}} \phi^{\mathrm{k}-1}(0)\right] \\
& +\mathbf{N}^{+}\left[\frac{\mathrm{u}^{\mathrm{n}}}{\mathrm{s}^{\mathrm{n}}} \mathbf{N}^{+}[\mathrm{R}(\phi(\mathrm{t} ; \mathrm{q}))+\mathrm{F}(\phi(\mathrm{t} ; \mathrm{q}))-\mathrm{g}(\mathrm{t})],\right. \\
\Longrightarrow & \mathrm{N}^{+}\left[\sum_{\mathrm{m}=1}^{\infty}\left[v_{\mathrm{m}-1}(\mathrm{t})+v_{\mathrm{m}-1}(\mathrm{at})\right]^{(\mathrm{n})}+\mathrm{F}\left(\sum_{\mathrm{m}=1}^{\infty} v_{\mathrm{m}-1}(\mathrm{t})\right)\right. \\
& \left.+\mathrm{R}\left(\sum_{\mathrm{m}=1}^{\infty} v_{\mathrm{m}-1}(\mathrm{t})\right)-\mathrm{g}\left(\sum_{\mathrm{m}=1}^{\infty} v_{\mathrm{m}-1}(\mathrm{t})\right)\right)=0 \\
\Longrightarrow & \mathrm{N}^{+}\left[\sum_{\mathrm{m}=0}^{\infty}\left[v_{\mathrm{m}}(\mathrm{t})+v_{\mathrm{m}}(\mathrm{at})\right]^{(\mathrm{n})}+\mathrm{F}\left(\sum_{\mathrm{m}=0}^{\infty} v_{\mathrm{m}}(\mathrm{t})\right)\right] \\
& +\mathrm{N}^{+}\left[\mathrm{R}\left(\sum_{\mathrm{m}=0}^{\infty} v_{\mathrm{m}}(\mathrm{t})\right)-\mathrm{g}\left(\sum_{\mathrm{m}=1}^{\infty} v_{\mathrm{m}-1}(\mathrm{t})\right)\right]=0 .
\end{aligned}
$$

Now take the inverse natural transform of both sides of (4.4) we obtain

$$
\mathrm{L}[\mathrm{S}(\mathrm{t})+\mathrm{S}(\mathrm{at})]+\mathrm{R}[\mathrm{S}(\mathrm{t})]+\mathrm{F}[\mathrm{S}(\mathrm{t})]-\mathrm{g}[\mathrm{S}(\mathrm{t})]=0 .
$$

And the result follows immediately. The proof for Case II (constant delay) is of the same process with that of Case I. 
Theorem 4.2. Suppose the solution components $v_{\mathrm{m}}(\mathrm{t})$ is defined in a Banach space $(\mathrm{C}[\mathrm{D}],\|\cdot\|)$, and let $\delta=$ $\left(\alpha_{2}+\alpha\right) \mathrm{d}$ for some $\delta \in(0,1)$ such that $\left\|v_{\mathrm{m}}\right\| \leqslant \delta\left\|v_{\mathrm{m}-1}\right\|$. Then the series solution (3.13) converges uniformly to the unique solution $v(t)$ of (3.1) and (3.2).

Proof. Let $\left\{S_{n}\right\}$ be a sequence of partial sum of the series (3.13). Then we need to show that $\left\{s_{n}\right\}_{\mathfrak{n}=0}^{\infty}$ is a Cauchy sequence in Banach space $(\mathrm{C}[\mathrm{D}],\|\cdot\|)$. So consider

$$
\left\|S_{m}-S_{m-1}\right\|=\left\|v_{m}\right\| \leqslant \delta\left\|v_{m-1}\right\| \leqslant \delta^{2}\left\|v_{m-2}\right\| \leqslant \cdots \leqslant \delta^{m}\left\|v_{0}\right\|
$$

Now for every $m, n \in N$ with $m \geqslant n$, then by the means of (4.5) and using triangle inequality we can successively obtain

$$
\begin{aligned}
\left\|S_{m}-S_{n}\right\| & =\left\|\left(S_{m}-S_{m-1}\right)+\left(S_{m-1}-S_{m-2}\right)+\cdots+\left(S_{n+1}-S_{n}\right)\right\| \\
& \leqslant\left\|S_{m}-S_{m-1}\right\|+\left\|S_{m-1}-S_{m-2}\right\| \cdots+\left\|S_{n+1}-S_{n}\right\| \\
& \leqslant \delta^{m}\left\|v_{0}(t)\right\|+\delta^{m-1}\left\|v_{0}(t)\right\|+\cdots+\delta^{n+1}\left\|v_{0}(t)\right\| \\
& =\delta^{n+1}\left\|v_{0}(t)\right\| \sum_{k=0}^{m-n} \delta^{k} \leqslant \delta^{n}\left\|v_{0}(t)\right\| \sum_{k=0}^{\infty} \delta^{k}=\delta^{n}\left\|v_{0}(t)\right\|\left(\frac{1}{1-\delta}\right) .
\end{aligned}
$$

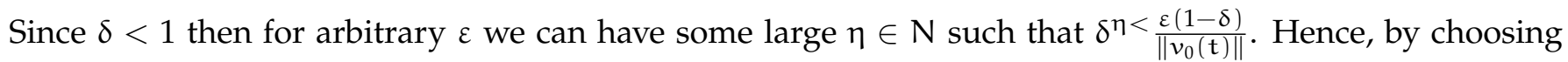
$n, m>N$ we obtain

$$
\left\|S_{m}-S_{n}\right\| \leqslant \delta^{n}\left\|v_{0}(t)\right\|\left(\frac{1}{1-\delta}\right)<\frac{\varepsilon(1-\delta)}{\left\|v_{0}(t)\right\|}\left\|v_{0}(t)\right\|\left(\frac{1}{1-\delta}\right)=\varepsilon
$$

Therefore, (4.7) shows that $\left\{s_{\mathfrak{n}}\right\}_{\mathfrak{n}=0}^{\infty}$ is a Cauchy sequence in Banach space $(C[D],\|\|$.$) , so the sequence$ converges, and hence the series solution in (3.13) also converges. Therefore, the proof is completed.

To show the uniqueness of this solution, suppose that $v(t)$ and $u(t)$ are two distinct solutions of equations (3.1) and (3.2). Now from (3.3) we have

$$
v(\mathrm{t})+v_{\beta}=\mathrm{L}^{-1}[\mathrm{~g}(\mathrm{t})-\mathrm{R}(v)-\mathrm{F}(v)],
$$

where $\mathrm{L}^{-1}$ is an inverse operator defined by $\int_{0}^{t}() d$.$t . Since v(t)$ and $u(t)$ are distinct solutions of Equations (3.1) and (3.2). So from (4.8) we obtain the following equation

$$
\begin{aligned}
\left\|(v-u)+\left(v_{\beta}-u_{\beta}\right)\right\| \leqslant\|(v-u)\| & =\left\|-\int_{0}^{t}[R(v-u)+F(v-u)] d t\right\| \\
& \leqslant \int_{0}^{t}[\|R(v-u)\|+\|F(v)-F(u)\|] d t \\
& \leqslant\left(\alpha_{2}\|v-u\|+\alpha\|v-u\|\right) d \leqslant \delta\|v-u\| .
\end{aligned}
$$

From (4.9) we obtained $(1-\delta)\|v-u\| \leqslant 0$ and since $\delta \in(0,1)$ then $\|v-u\| \leqslant 0$ implies that $v=u$ and hence the proof.

Theorem 4.3. If the series solution (3.13) Converges then the maximum absolute error between the series and solution $v(\mathrm{t})$ to problem (3.1) and (3.2) is estimated to be

$$
\left\|v(t)-\sum_{m=0}^{M} v_{m}(t)\right\| \leqslant \frac{\delta^{n}}{(1-\delta)}\left\|v_{0}(t)\right\| .
$$


Proof. From Theorem 4.2 and (4.6) we have

$$
\left\|S_{m}-S_{n}\right\| \leqslant \frac{\delta^{n}}{(1-\delta)}\left\|v_{0}(t)\right\| .
$$

So as $m \rightarrow \infty$ then $S_{m} \rightarrow v(t)$, hence we have

$$
\left\|v(t)-S_{n}\right\| \leqslant \frac{\delta^{n}}{(1-\delta)}\left\|v_{0}(t)\right\| .
$$

Since $\delta \in(0,1)$ then $1-\delta \leqslant 1$ and from (4.10) we obtain

$$
\left\|v(t)-\sum_{m=0}^{M} v_{m}\right\| \leqslant \frac{\delta^{n}}{(1-\delta)}\left\|v_{0}(t)\right\| .
$$

Hence the proof is completed.

\section{Analytical examples}

In this section, Algorithm (3.14) will be applied to obtain solutions of some linear and nonlinear DDEs with both proportional and constant delays.

Example 5.1 ([26]). Consider the following first order linear NDDE with proportional delay

$$
v^{\prime}(\mathrm{t})-\frac{1}{2} v^{\prime}\left(\frac{\mathrm{t}}{2}\right)+v(\mathrm{t})-\frac{1}{2} v\left(\frac{\mathrm{t}}{2}\right)=0,0 \leqslant \mathrm{t} \leqslant 1, \quad v(0)=1 .
$$

Equation (5.1) has an exact solution $e^{-t}[26]$.

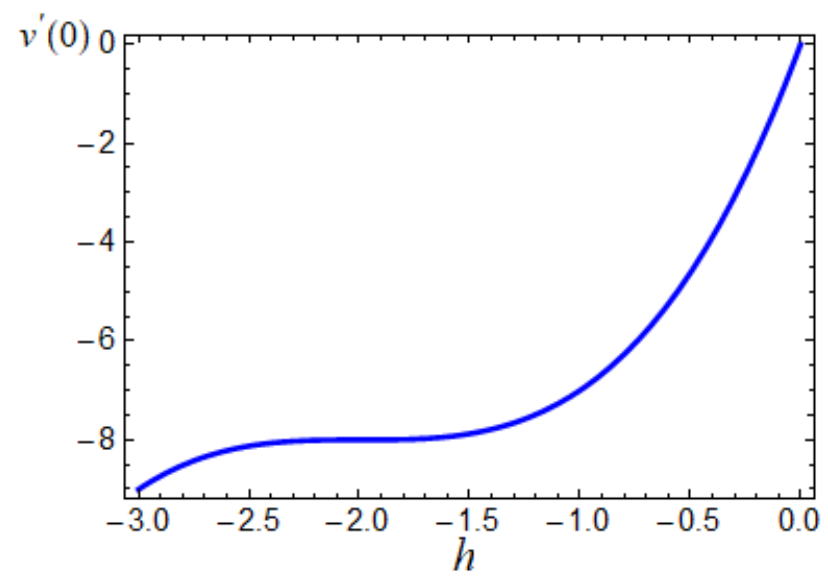

Figure 1: The h-curve for the third order approximation of Example 5.1.

Taking the natural transform of both side of (5.1) we obtain

$$
\mathbf{N}^{+}\left[v(\mathrm{t})-\frac{1}{2} v\left(\frac{\mathrm{t}}{2}\right)\right]+\frac{\mathrm{u}}{\mathrm{s}} \mathbf{N}^{+}\left[v(\mathrm{t})-\frac{1}{2} v\left(\frac{\mathrm{t}}{2}\right)\right]=0 .
$$

From (5.2) we can define a nonlinear operator,

$$
\mathrm{N}[\phi(\mathrm{t} ; \mathrm{q})]=\mathbf{N}^{+}\left[\phi(\mathrm{t} ; \mathrm{q})-\frac{1}{2} \phi\left(\frac{\mathrm{t}}{2} ; \mathrm{q}\right)\right]+\frac{\mathrm{u}}{\mathrm{s}} \mathbf{N}^{+}\left[\phi(\mathrm{t} ; \mathrm{q})-\frac{1}{2} \phi\left(\frac{\mathrm{t}}{2} ; \mathrm{q}\right)\right] .
$$


Now using (3.14) the recursive relation of (5.1) can be obtained as

$$
v_{m}(t)=\left(\chi_{m}+h\right) v_{m-1}(t)-h v_{m-1}\left(\frac{t}{2}\right)+h \mathbf{N}^{-}\left\{\frac{u}{s} \mathbf{N}^{+}\left[R\left(v_{m-1}(t)\right)\right]\right\}, m \geqslant 1 .
$$

By choosing an initial approximation $v_{0}(t)=1$ and using (5.3) we obtained the following components as

$$
\begin{aligned}
& v_{1}(t)=\frac{1}{2} h t \\
& v_{2}(t)=\frac{1}{4}\left(h^{2}+2 h\right) t+\frac{3}{16} h^{2} t^{2} \\
& v_{3}(t)=\frac{1}{8}\left(h^{3}+4 h^{2}+4 h\right) t+\frac{1}{64}\left(15 h^{3}+24 h^{2}\right) t^{2}+\frac{7}{128} h^{3} t^{3} .
\end{aligned}
$$

The remaining terms of $v_{m}(t)$ for $m \geqslant 3$ can be obtained in a similar way.

Now, from Fig. 1 the optimal value of $h=-2$. Hence, the series solution for the fifth order approximation of (5.1) is given as

$$
v(t)=1-t+\frac{t^{2}}{2 !}-\frac{t^{3}}{3 !}+\frac{t^{4}}{4 !}-\frac{t^{5}}{5 !}+\cdots,
$$

which converges to exact solution $e^{-t}$ of the given problem. Therefore, the fifth order approximation of the algorithm (3.14) converged to the exact solution of problem (5.1). This reduces the computational size used in [26] to obtain the approximate solution of the problem. The graph of the exact solution and fifth order approximate solution is plotted in Fig. 2.

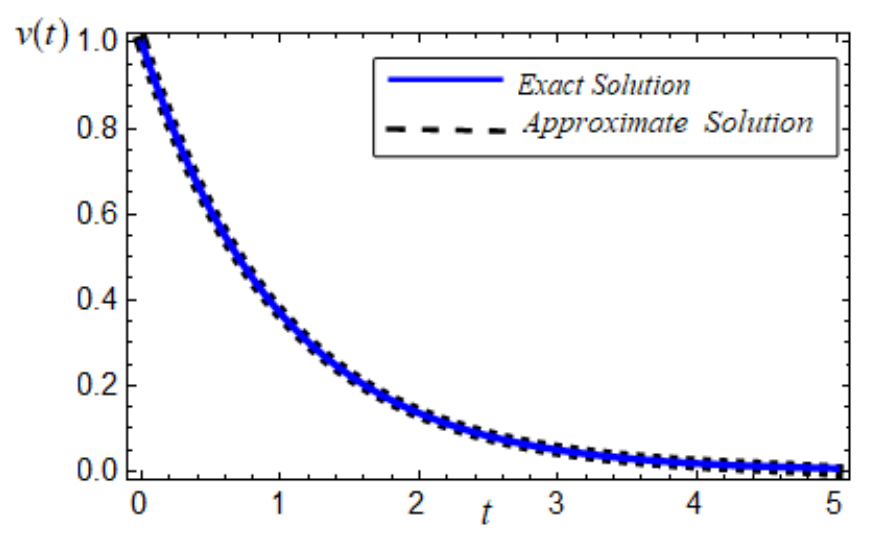

Figure 2: The exact and approximate solution of Example 5.1.

Example 5.2 ([9]). Consider the following first order linear NDDE with constant delay

$$
v^{\prime}(\mathrm{t})+\frac{1}{4} v^{\prime}(\mathrm{t}-1)-v(\mathrm{t})-v(\mathrm{t}-1)=0,0 \leqslant \mathrm{t} \leqslant 1, v(\mathrm{t})=-\mathrm{t}, \mathrm{t} \in[-1,0] .
$$

Taking the natural transform of both side of (5.4) we obtain

$$
\mathbf{N}^{+}\left[v(t)+\frac{1}{4} e^{-\frac{s}{u}} v(t)\right]-\frac{1}{4 s}-\frac{u}{s} \mathbf{N}^{+}[v(t)+v(t-1)]=0
$$

From (5.5) we can define a nonlinear operator,

$$
\mathrm{N}[\phi(\mathrm{t} ; \mathrm{q})]=\mathbf{N}^{+}\left[\phi(\mathrm{t} ; \mathrm{q})+\frac{1}{4} e^{-\frac{\mathrm{s}}{\mathrm{u}}} \phi(\mathrm{t} ; \mathrm{q})\right]-\frac{1}{4 \mathrm{~s}}-\frac{\mathrm{u}}{\mathrm{s}} \mathbf{N}^{+}[\phi(\mathrm{t} ; \mathrm{q})+\phi(\mathrm{t}-1 ; \mathrm{q})] .
$$


Now using (3.14) the recursive relation of Example 5.2 can be obtained as

$$
v_{m}(t)=\left(\chi_{m}+h\right) v_{m-1}(t)+\frac{1}{4} h v_{m-1}(t-1)-\frac{1}{4}\left(1-\chi_{m}\right) h \mathbf{N}^{-}\left[\frac{1}{s}\right]-h \mathbf{N}^{-}\left\{\frac{u}{s} \mathbf{N}^{+}\left[R\left(v_{m-1}(t)\right)\right]\right\}, m \geqslant 1 .
$$

By choosing an initial approximation $v_{0}(t)=-t$ and using (5.6) we obtained the following components

$$
\begin{aligned}
& v_{1}(t)=h t^{2}-\frac{9}{4} h t \\
& v_{2}(t)=-\frac{2}{3} h^{2} t^{3}+\frac{1}{8}\left(14 h^{2}+5 h\right) t^{2}-\frac{9}{16}\left(4 h^{2}+4 h\right) \\
& v_{3}(t)=\frac{1}{3} h^{3} t^{4}-\frac{1}{24}\left(24 h^{3}+7 h^{2}\right) t^{3}+\frac{1}{16}\left(71 h^{3}+81 h^{2}+10 h\right) t^{2}-\frac{9}{16}\left(5 h^{3}+9 h^{2}+4 h\right) t .
\end{aligned}
$$

The remaining terms of $v_{m}(t)$ for $m \geqslant 3$ can be obtained in a similarly. Therefore, from Fig. 3 the optimal value of $h$ can be choose as -1 and the solution of Example 5.2 can be expressed in a series form as

$$
v(t)=\frac{5 t}{4}+\frac{t^{2}}{8}+\frac{t^{3}}{24}+\frac{t^{4}}{96}+\frac{t^{5}}{480}+\cdots=t-\frac{1}{4}+\frac{1}{4}\left(1+t+\frac{t^{2}}{2 !}+\frac{t^{3}}{3 !}+\frac{t^{4}}{4 !}+\frac{t^{5}}{5 !}+\ldots\right) .
$$

Equation (5.7) gives the Taylor series expansion of the exact solution of the given problem, i.e.,

$$
v(\mathrm{t})=\mathrm{t}-\frac{1}{4}+\frac{1}{4} \mathrm{e}^{\mathrm{t}}
$$

Fabiano [9] obtained only the approximate solution of (5.4), and by using our approach the closed form solution of this problem was successfully obtained.

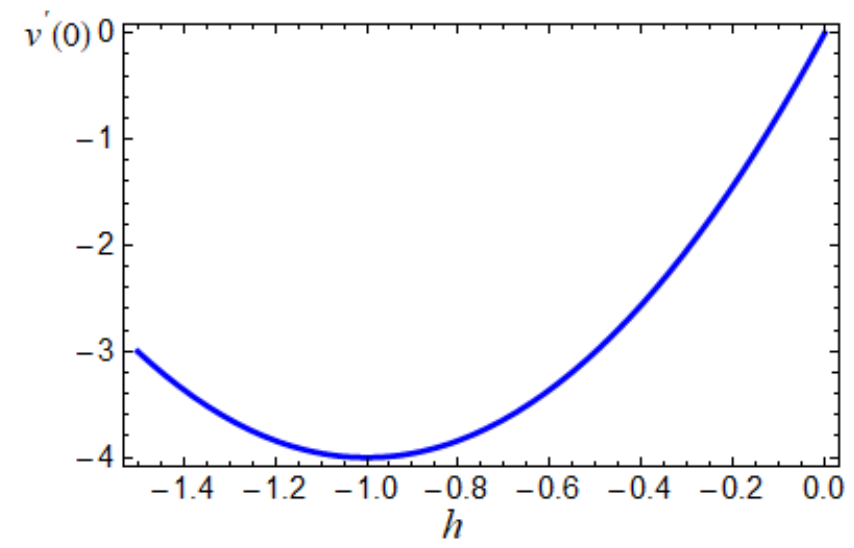

Figure 3: The h-curve for the third order approximation of Example 5.2.

Example 5.3 ([8]). Consider the third order nonlinear NDDE with proportional delay

$$
v^{\prime \prime \prime}(\mathrm{t})-\frac{1}{2} v^{\prime \prime \prime}\left(\frac{\mathrm{t}}{4}\right)-v^{\prime \prime}\left(\frac{\mathrm{t}}{3}\right)-v^{\prime}\left(\frac{\mathrm{t}}{2}\right)-(v(\mathrm{t}))^{2}-\mathrm{g}(\mathrm{t})=0,0 \leqslant \mathrm{t} \leqslant 1,
$$

where

$$
\begin{aligned}
g(t)= & \frac{e^{\frac{t}{12}}}{54}\left\{-13752 \sin (2 t)-54 e^{\frac{7 t}{12}} \cos ^{2}(8 t)+1727 \cos (2 t)+6 e^{\frac{t}{36}}\left(48 \sin \left(\frac{8 t}{3}\right)+575 \cos \left(\frac{8 t}{3}\right)\right)\right. \\
& \left.-18 e^{\frac{t}{12}}(\cos (4 t)-24 \sin (4 t))+2 e^{\frac{t}{4}}(13752 \sin (8 t)-1727 \cos (8 t))\right\}
\end{aligned}
$$


with the given initial condition

$$
v(0)=1, v^{\prime}(0)=\frac{1}{3}, v^{\prime \prime}(0)=-\frac{575}{9} .
$$

Taking the natural transform of both sides of (5.8) we obtained

$$
\mathbf{N}^{+}\left[v(t)-32 v\left(\frac{t}{4}\right)\right]+\left[\frac{41}{s}+\frac{41 u}{3 s^{2}}-\frac{23575 u^{2}}{9 s^{3}}\right]-\frac{u^{3}}{s^{3}} \mathbf{N}^{+}\left[v^{\prime \prime}\left(\frac{t}{3}\right)+v^{\prime}\left(\frac{t}{2}\right)+(v(t))^{2}\right]=0 .
$$

From (5.9) we can define a nonlinear operator,

$$
\begin{aligned}
\mathrm{N}[\phi(\mathrm{t} ; \mathrm{q})]= & \mathbf{N}^{+}\left[\phi(\mathrm{t} ; \mathrm{q})-32 \phi\left(\frac{\mathrm{t}}{4} ; \mathrm{q}\right)\right]+\left[\frac{41}{\mathrm{~s}}+\frac{41 \mathrm{u}}{3 \mathrm{~s}^{2}}-\frac{23575 \mathrm{u}^{2}}{9 \mathrm{~s}^{3}}\right] \\
& -\frac{\mathrm{u}^{3}}{\mathrm{~s}^{3}} \mathbf{N}^{+}\left[\phi^{\prime \prime}\left(\frac{\mathrm{t}}{3} ; \mathrm{q}\right)+\phi^{\prime}\left(\frac{\mathrm{t} ;}{2} ; \mathrm{q}\right)+(\phi(\mathrm{t} ; \mathrm{q}))^{2}\right] .
\end{aligned}
$$

Now using (3.14) the recursive relation of Example 5.3 can be obtained as

$$
\begin{aligned}
v_{m}(t)= & \left(\chi_{m}+h\right) v_{m-1}(t)-32 h v_{m-1}\left(\frac{t}{4}\right)+h\left(1-\chi_{m}\right) \mathbf{N}^{-}\left[\frac{41}{s}+\frac{41 u}{3 s^{2}}-\frac{23575 u^{2}}{9 s^{3}}\right] \\
& -h \mathbf{N}^{-}\left\{\frac{u^{3}}{s^{3}} \mathbf{N}^{+}\left[R\left(v_{m-1}(t)\right)+H_{m-1}\left(v_{0}, v_{1}, \ldots, v_{n}\right)+g(t)\right]\right\}, m \geqslant 1 .
\end{aligned}
$$

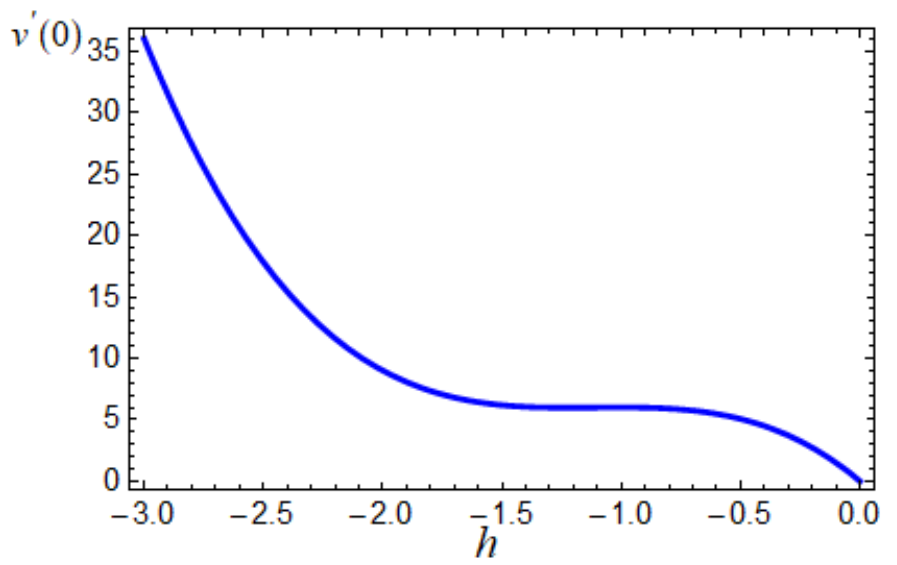

Figure 4: The h-curve for the third order approximation of Example 5.3.

By choosing an initial approximation $v_{0}(t)=1+\frac{t}{3}-\frac{575 t^{2}}{18}$ and (5.10) we obtained the following components as

$$
\begin{aligned}
& v_{1}(t)=-\frac{2312063}{15552} h t^{4}+\frac{1727}{324} h t^{3}-\frac{11500}{9} h t^{2}+\frac{34}{3} h t, \\
& v_{2}(t)=(1+h) v_{1}(t)-32 v_{1}\left(\frac{t}{4}\right)-h \mathbf{N}^{-}\left\{\frac{u^{3}}{s^{3}} \mathbf{N}^{+}\left[R\left(v_{1}(t)\right)+H_{1}\left(v_{0}, v_{1}, \ldots, v_{n}\right)+g(t)\right]\right\}, \\
& v_{3}(t)=(1+h) v_{2}(t)-32 v_{2}\left(\frac{t}{4}\right)-h N^{-}\left\{\frac{u^{3}}{s^{3}} N^{+}\left[R\left(v_{2}(t)\right)+H_{2}\left(v_{0}, v_{1}, \ldots, v_{n}\right)+g(t)\right]\right\} .
\end{aligned}
$$

Therefore, by choosing the optimal value of $h=-1$ from Fig. 4, then third order approximate solution of Example 5.3 can be obtained in a series form as

$$
v(t)=1+\frac{t}{3}-\frac{575 t^{2}}{18}-\frac{1727}{162} t^{3}+\frac{328321}{1944} t^{4}+\frac{1653121}{29160} t^{5}+\cdots,
$$


which converges to Taylor series expansion of $e^{\frac{t}{3}} \cos (8 t)$. Therefore, using the derived method (3.14) we were able to obtain a closed form solution of Example 5.3 from only three iterations. Unlike in the case of Bhrawey et al. [8] only the approximate solution of the problem was able to obtain. The graph of the exact solution and the third order approximate solution is plotted in Fig. 5.

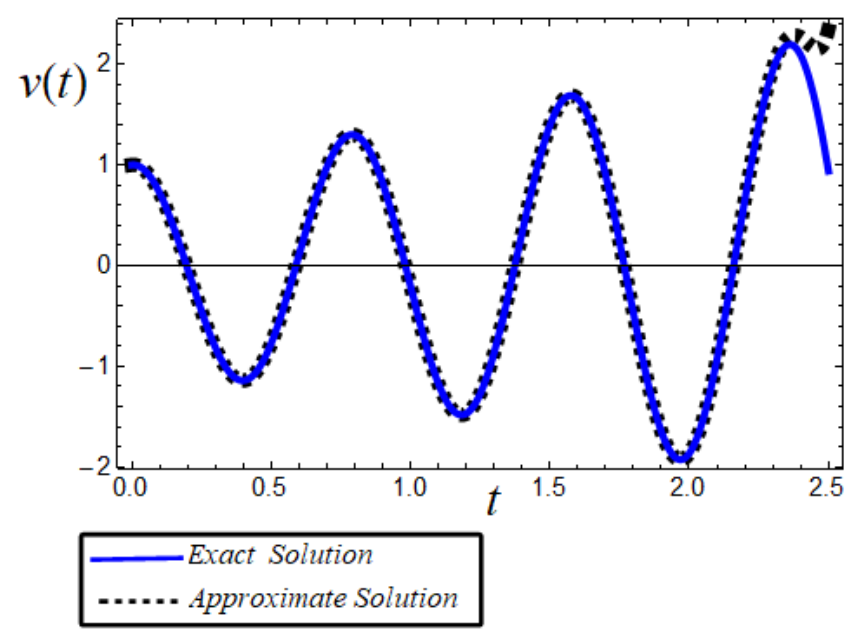

Figure 5: The exact and approximate solution of Example 5.3.

Example 5.4 ([28]). Finally we consider the following Cauchy problem (nonlinear Ricatti equation)

$$
v^{\prime}(t-2)-t^{2} v(2 t-3)-v^{2}(t-1)+5 t^{4}-20 t^{3}+19 t^{2}-2 t-3=0, \quad x(0)=-2 .
$$

The exact solution to this problem is $v(t)=t^{2}-t-2$ [28]. Taking the natural transform of both side of (5.11) we obtain

$$
e^{-\frac{2 s}{u}} \mathbf{N}^{+}[v(t)]+\left[\frac{2}{s}-\frac{3 u}{s^{2}}\right]-\frac{u}{s} \mathbf{N}^{+}\left[t^{2} v(2 t-3)+v^{2}(t-1)-\left(5 t^{4}-20 t^{3}+19 t^{2}-2 t\right)\right]=0 .
$$

From (5.12) we can define a nonlinear operator

$$
\begin{aligned}
\mathrm{N}[\phi(t ; q)]= & e^{-\frac{2 s}{u}} \mathbf{N}^{+}[\phi(t ; q)]+\left[\frac{2}{s}-\frac{3 u}{s^{2}}\right]-\frac{u}{s} \mathbf{N}^{+}\left[t^{2} \phi(2 t-3 ; q)\right. \\
& \left.+\phi^{2}(t-1 ; q)-\left(5 t^{4}-20 t^{3}+19 t^{2}-2 t\right)\right] .
\end{aligned}
$$

Now using (3.14) the recursive relation of Example 5.4 can be obtained as

$$
\begin{aligned}
v_{m}(t)= & x_{m} v_{m-1}(t)+h v_{m-1}(t-2)-h\left(1-\chi_{m}\right) \mathbf{N}^{-}\left[\frac{3 u}{s^{2}}-\frac{2}{s}\right] \\
& -h N^{-}\left\{\frac{u}{s} \mathbf{N}^{+}\left[R\left(v_{m-1}(t)\right)+H_{m-1}\left(v_{0}, v_{1}, \ldots, v_{n}\right)-g(t)\right]\right\}, m \geqslant 1 .
\end{aligned}
$$

By choosing an initial approximation $v_{0}(t)=t^{2}-t-2$ and using (5.13) we obtain the following components as

$$
\begin{aligned}
v_{1}(t)= & t^{2}-(8 h+1) t+(6 h-2), \\
v_{2}(t)= & \frac{1}{4}\left(24 h^{2}+25 h\right) t^{4}-\frac{1}{3}\left(106 h^{2}+28 h\right) t^{3}-\left(42 h^{2}-h+1\right) t^{2} \\
& -\left(8 h^{2}+13 h-1\right) t-\left(22 h^{2}+6 h-2\right) .
\end{aligned}
$$


It is easy to see that at $h=0$ the second order approximate solution gives the actual exact solution of the problem, i.e.,

$$
v(t)=v_{0}(t)+v_{1}(t)+v_{2}(t)=t^{2}-t-2
$$

In [28] Yuzbasi was only able to compute approximate numerical solution of this problem of the form

$$
v(t)=\left(0.1 \times 10^{-19}\right) t^{3}+t^{2}-t-2 .
$$

\section{Conclusion}

In this research, the combination of HAM and natural transform yield a reliable and efficient analytical approach suitable for solving linear and nonlinear NDDEs with proportional and constants delays. The He's polynomial is utilized to generate the series of nonlinear terms of NDDEs. In order to show the efficiency of our derived algorithms over the reference methods, solutions to different problems were obtained in a series form which converge to the exact solution with high precision using few number of iterations. Therefore, the presented technique is effective and straightforward for analytic treatment of Linear and nonlinear NDDEs which is also capable of solving other forms of linear and nonlinear problems.

\section{Acknowledgment}

The authors like express their gratitude for financial support from Transdisciplinary Research Grant Scheme (TRGS) under the research program of Characterization of Spatio-temporal Marine Microalgae Ecological Impact Using Multi-sensor Over Malaysia Waters for the specific sub-project of Mathematical Modeling of Harmful Algal Blooms (HABs) in Malaysia Waters (R.J130000.7809.4L854) and Fundamental Research Grant Scheme (FRGS) of titled Tumor-Hyperthermia Immunotherapy Model with Optimal Temperature Control Strategy (5F220) funded by the Ministry of Education, Malaysia. Authors also like to appreciate the Universiti Teknologi Malaysia for providing all the necessary support and facilities toward the success of this research.

\section{References}

[1] A. K. Alomari, M. S. M. Noorani, R. Nazar, Solution of delay differential equation by means of homotopy analysis method, Acta Appl. Math., 108 (2009), 395-412. 1

[2] K. Barzinji, N. Maan, N. Aris, Fuzzy delay predator-prey system: existence theorem and oscillation property of solution, Int. J. Math. Anal. (Ruse), 8 (2014), 829-847. 1

[3] J. J. Batzel, T. H. Tran, Stability of the human respiratory control system I. Analysis of a two-dimensional delay state-space model, J. Math. Biol., 41 (2000), 45-79. 1

[4] F. B. M. Belgacem, R. Silambarasan, Advances in the natural transform, AIP conference proceedings, 2012 (2012), 106-110. 2, 2.2, 2.4

[5] F. B. M. Belgacem, R. Silambarasan, Maxwell's equations solutions by means of the natural transform, Math. Eng. Sci. Aerosp., 3 (2012), 313-323. 2.3, 2

[6] F. B. M. Belgacem, R. Silambarasan, Theory of Natural Transform, Math. Eng. Sci. Aerosp., 3 (2012), 99-124. 2.1, 2, 2.1

[7] A. Bellen, M. Zennaro, Numerical methods for delay differential equations, Oxford University Press, New York, (2003). 1

[8] A. H. Bhrawy, M. A. Alghamdi, D. Baleanu, Numerical solution of a class of functional-differential equations using Jacobi pseudospectral method, Abstr. Appl. Anal., 2013 (2013), 9 pages. 1, 5.3, 5.3

[9] R. H. Fabiano, A semidiscrete approximation scheme for neutral delay-differential equations, Int. J. Numer. Anal. Model., 10 (2013), 712-726. 5.2, 5

[10] Z. H. Khan, W. A. Khan N-transform properties and applications, NUST J. Eng. Sci., 1 (2008), 127-133. 2

[11] S.-J. Liao, The proposed homotopy analysis technique for the solution of nonlinear problems, Shanghai Jiao Tong University (Ph. D. Thesis), Shanghai, (1992). 1

[12] S.-J. Liao, K. F. Cheung, Homotopy analysis of nonlinear progressive waves in deep water, J. Engrg. Math., 45 (2003), 105-116. 1 
[13] S.-J. Liao, J. Su, A. T. Chwang, Series solutions for a nonlinear model of combined convective and radiative cooling of a spherical body, Int. J. Heat Mass Transfer, 49 (2006), 2437-2445. 1

[14] L. P. Liu, T. Kalmár-Nagy, High-dimensional harmonic balance analysis for second-order delay-differential equations, J. Vib. Control, 16 (2010), 1189-1208. 1

[15] D. Loonker, P. K. Banerji, Natural transform and solution of integral equations for distribution spaces, American J. Math. Sci., 3 (2014), 65-72. 2

[16] N. Maan, K. Barzinji, N. Aris, Fuzzy delay differential equation in predator-prey interaction: analysis on stability of steady state, Proceedings of the World Congress on Engineering, 2013 (2013), 3-5. 1

[17] S. Maitama, An efficient technique for solving linear and nonlinear fractional partial differential equations, Math. Eng. Sci. Aerosp., 8 (2017), 521-534. 2

[18] S. Maitama, I. Abdullahi, A new analytical method for solving linear and nonlinear fractional partial differential equations, Prog. Fract. Differ. Appl., 2 (2016), 247-256. 2

[19] S. Maitama, W. D. Zhao, New homotopy analysis transform method for solving multidimensional fractional diffusion equations, Arab J. Basic Appl. Sci., 27 (2020), 27-44. 1

[20] L. Muhsen, N. Maan, Modeling of Human Postural Balance Using Neutral Delay Differential Equation to Solvable Lie Algebra Classification, Life Sci. J., 11 (2014), 1145-1152. 1

[21] L. Muhsen, N. Maan, Lie group analysis of second-order non-linear neutral delay differential equations, Malaysian J. Math. Sci., 10 (2016), 117-129. 1

[22] Z. Odibat, S. Momani, H. Xu, A reliable algorithm of homotopy analysis method for solving nonlinear fractional differential equations, Appl. Math. Model., 34 (2010), 593-600. 1

[23] M. S. Rawashdeh, S. Maitama, Solving nonlinear ordinary differential equations using the NDM, J. Appl. Anal. Comput., 5 (2015), 77-88. 2, 2

[24] F. A. Rihan, D. H. Abdel Rahman, S. Lakshmanan, A. S. Alkhajeh, A time delay model of tumour-immune system interactions: Global dynamics, parameter estimation, sensitivity analysis, Appl. Math. Comput., 232 (2014), 606-623. 1

[25] F. A. Rihan, A. A. Azamov, H. J. Al-Sakaji, An Inverse problem for delay differential equations: parameter estimation, nonlinearity, sensitivity, Appl. Math. Inf. Sci., 12 (2018), 63-74. 1

[26] M. G. Sakar, Numerical solution of neutral functional-differential equations with proportional delays, Int. J. Optim. Control. Theor. Appl. IJOCTA, 7 (2017), 186-194. 1, 5.1, 5, 5

[27] S. K. Vanani, A. Aminataei, On the numerical solution of neutral delay differential equations using multiquadric approximation scheme, Bull. Korean Math. Soc., 45 (2008), 663-670. 1

[28] S. Yüzbaş1, A numerical approximation based on the Bessel functions of first kind for solutions of Riccati type differentialdifference equations, Comput. Math. Appl., 64 (2012), 1691-1705. 5.4, 5, 5

[29] J. J. Zhao, Y. Cao, Y. Xu, Legendre-Gauss collocation methods for nonlinear neutral delay differential equations, Adv. Difference Equ., 2015 (2015), 24 pages. 1 